\title{
Octupole correlations in positive-parity states of rare-earth and actinide nu- clei
}

\author{
M. Spieker ${ }^{1, a}$, S. Pascu ${ }^{1,2}$, D. Bucurescu², T. Faestermann ${ }^{3}$, R. Hertenberger ${ }^{4}$, H.-F. Wirth ${ }^{4}$, N.-V. Zamfir ${ }^{2}$, and \\ A. Zilges ${ }^{1}$ \\ ${ }^{1}$ Institut für Kernphysik, Universität zu Köln, D-50937 Köln, Germany \\ ${ }^{2}$ Horia Hulubei National Institute of Physics and Nuclear Engineering, Bucharest, Romania \\ ${ }^{3}$ Physik Department, Technische Universität München, München, Germany \\ ${ }^{4}$ Fakultät für Physik, Ludwig-Maximilians Universität München, München, Germany
}

\begin{abstract}
In this contribution, further evidence of the importance of multiphonon-octupole excitations to describe experimental data in the rare earths and actinides will be presented. First, new results of a $(p, t)$ experiment at the Q3D magnetic spectrograph in Munich will be discussed, which was performed to selectively excite $J^{\pi}=0^{+}$states in ${ }^{240} \mathrm{Pu}$. spdf interacting boson model (IBM) calculations suggest that the previously proposed double-octupole phonon nature of the $J^{\pi}=0_{2}^{+}$state is not in conflict with its strong $(p, t)$ population. Second, the framework of the IBM has been adopted for the description of experimental observables related to octupole excitations in the rare earths. Here, the IBM is able to describe the signature splitting for positiveand negative-parity states when multi-dipole and multi-octupole bosons are included. The present study might support the idea of octupole-phonon condensation at intermediate spin $\left(J^{\pi}=10^{+}\right)$leading to the change in yrast structure observed in ${ }^{146} \mathrm{Nd}$.
\end{abstract}

\section{Introduction}

Strong octupole correlations are observed in rare-earth and actinide nuclei owing the fact that the Fermi surface for both neutrons and protons lies between single-particle orbitals which differ by $\Delta j=\Delta l=3$ [1]. One of the main features are sequences of low-lying negative-parity states in deformed nuclei $\left(E_{x} \leq 1 \mathrm{MeV}\right)$. If the octupole correlations are particulary strong, octupole deformation might already be realized at quite low spin indicated by the observation of an alternating-parity band. Only recently, the expected strong $E 3$ ground-state transition from the $3^{-}$state and the onset of an alternating-parity band at $J \approx 5$ have been observed for ${ }^{224} \mathrm{Ra}$ [2]. The possibility of multiphonon-octupole states due to the lowlying one-phonon states was discussed decades ago but new interest was triggered by state-of-the-art experiments and refined theoretical approaches. In ${ }^{240} \mathrm{Pu}$, an excited $K^{\pi}=0^{+}$rotational band was observed decaying exclusively to the one-octupole phonon $K^{\pi}=0^{-}$band via enhanced $E 1$ transitions at high spin [3]. At the same time, an alternating-parity band with connecting enhanced $E 1$ transitions, i.e. several m.W.u., had been observed with the same setup for the ground-state and one-octupole phonon band starting at $J \approx 20[3,4]$. Two different theoretical approaches were presented to explain the experimental observations $[3,5]$, both pointing out the importance of including the octupole degree of freedom and predicting

\footnotetext{
a e-mail: spieker@ikp.uni-koeln.de
}

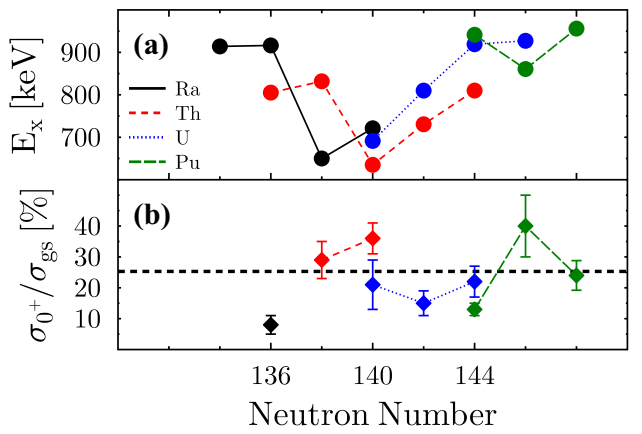

Figure 1. Properties of the $J^{\pi}=0_{2}^{+}$states in the actinides. (a) While there is a clear minimum in excitation energy around $N \sim$ 140 , (b) the relative transfer strength is uniformly strong with about $25 \%$ of the ground-state strength. Data taken from $[9,10]$.

the double-octupole nature of the excited $K^{\pi}=0^{+}$band in ${ }^{240} \mathrm{Pu}$. However, the nature of excited $J^{\pi}=0^{+}$states is a controversially discussed topic [6]. Especially in the actinides a pairing-isomeric character $[7,8]$ was proposed because of the uniformly strong $(p, t)$ population of the $J^{\pi}=0_{2}^{+}$states $[9,10]$, see Fig. 1 , and the comparably weak $(t, p)$ population observed for some nuclei [7].

State-of-the-art experimental setups, especially the Q3D magnetic spectrograph of the Maier-Leibnitz Laboratory (MLL) in Munich, delivered new high-quality data 
on a large number of monopole excitations in weakly and deformed rare earth and actinide nuclei by means of the $(p, t)$ reaction, see e.g., [11-13]. This new data allow to stringently test theoretical models, the nature of monopole excitations in nuclei, and the importance of including the octupole degree of freedom to describe these.

In this conference proceeding, results from a ${ }^{242} \mathrm{Pu}(p, t)^{240} \mathrm{Pu}$ experiment, obtained with the Q3D@MLL and already published in Ref. [13], will be shortly discussed focussing especially on the nature of the $J^{\pi}=0_{2,3}^{+}$states and their description in spdf interacting boson model (IBM) [14, 15]. This model had also predicted the double-dipole/octupole nature of some low-lying $0^{+}$states in the actinides [15]. To further test the robustness of this theoretical approach, ${ }^{146} \mathrm{Nd}$ has also been studied with the IBM since a very interesting change in yrast structure is observed for positive- and negativeparity states [16]. This observation will also be discussed along the lines of the condensation of rotational-aligned octupole phonons proposed in [17].

\section{The case of ${ }^{240} \mathrm{Pu}$}

${ }^{240} \mathrm{Pu}$ is a very interesting case because of the wealth of new experimental data obtained for the $K^{\pi}=0_{2}^{+}$rotational band with Gammasphere [3, 4] and at the Q3D in our recent experiment [13]. As mentioned above, this excited $K^{\pi}=0^{+}$band was proposed to be of double-octupole phonon nature. Previously, it had also been pointed out that the $K^{\pi}=0_{2}^{+}$and $K^{\pi}=0_{3}^{+}$states have very different structures, as indicated by their different $E 0$ decay behavior to the ground-state band [18]. To test if the strong $(p, t)$ population of the $J^{\pi}=0_{2}^{+}$state $[9,10]$ is in conflict with these observations and in particular with the doubleoctupole phonon structure, we have confronted the spdf IBM with our extended $(p, t)$ data up to $3 \mathrm{MeV}$ and also took a close look at the $E 0$ and $E 1$ decay behavior of the close-lying $0_{2}^{+}$and $0_{3}^{+}$states $(\Delta E=230 \mathrm{keV})$ [13].

The comparison of the IBM predictions to the experimental data for the summed relative transfer strength

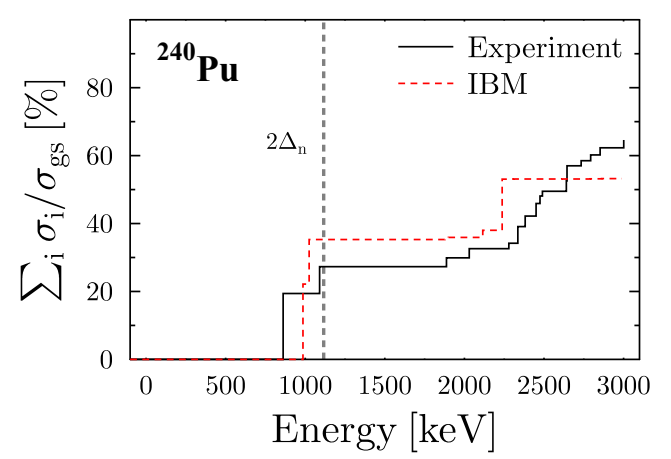

Figure 2. Summed relative transfer strength for $J^{\pi}=0^{+}$ states observed in ${ }^{242} \mathrm{Pu}(p, t){ }^{240} \mathrm{Pu}$ (solid line) in comparison with the predictions of the $s p d f$ IBM (red dashed line). The 2-quasiparticle energy $\left(2 \Delta_{\mathrm{n}} \approx 2 \mathrm{QP}\right)$ is presented as a shaded dashed line. $\sum \sigma_{\mathrm{i}} / \sigma_{\mathrm{gs}}$ is shown in Fig. 2. As in the experiment, two close-lying states are observed which lie below the 2quasiparticle energy (2QP) and might therefore be considered as being of collective origin. Their transfer strength is also found in good agreement with the experiment. Above this energy, the IBM is able to describe the correct transfer strength up to $2.5 \mathrm{MeV}$, using the transfer operator presented in [13]. It fails however to describe the correct fragmentation of the transfer strength. This is most likely due to the $2 \mathrm{QP}$ configurations which are not included in the model space of the IBM. The collective transfer strength, observed in ${ }^{232} \mathrm{U},{ }^{228,230} \mathrm{Th}$ [11], and ${ }^{240} \mathrm{Pu}$ [13] between $2-2.5 \mathrm{MeV}$, is apparently included in the model space of the spdf IBM and is connected to a higher-lying excited double-dipole/octupole phonon state.

As in experiment, the two lowest-lying $0^{+}$states are of different character. While the $0_{2}^{+}$state is predicted to be of dominant double-octupole phonon structure, the $0_{3}^{+}$is identfied to be a quadrupole excitation. As presented in our recent work [13], this interpretation is also able to describe the very different $\gamma$-decay behavior of the states including the less enhanced $E 0$ decay $0_{2}^{+} \rightarrow 0_{\mathrm{gs}}^{+}$and the enhanced experimental $B(E 1) / B(E 2)$ ratio of the $0_{2}^{+}$state of $13.7(3) \cdot 10^{-6} \mathrm{fm}^{-2}$ (IBM: $10.4 \cdot 10^{-6} \mathrm{fm}^{-2}$ ). The same predicted ratio is two orders of magnitude lower for the $0_{3}^{+}$ state and an $E 1$ decay has consequently not been observed yet. However, while the proposed double-octupole phonon structure is obviously not in conflict with the strong $(p, t)$ population of the $0_{2}^{+}$state in ${ }^{240} \mathrm{Pu}$, it has to be pointed out that it is not responsible for it. In fact, the inspection of the wave functions show that it is the $d$-boson content of the $0_{2}^{+}$wave function, which is causing the strong $(p, t)$ population. This also supports the importance of quadrupole pairing besides monopole pairing to describe the transfer strength [8].

\section{Multiple-octupole phonons in ${ }^{146} \mathrm{Nd}$}

Considering octupole correlations, ${ }^{146} \mathrm{Nd}$ seems to play a special role since two long alternating-parity bands are experimentally observed, which change yrast character [16]. Here, one can also look at the alignment $i_{\mathrm{x}}$ with the rotational axis as a function of rotational frequency $\hbar \omega$ for the four individual bands, which will be denoted in the following by GSB for the ground-state band, OCT for the one-octupole phonon band, $\pi=+$ and $\pi=-$ for the additional positive- and negative-parity band, respectively. The alignment has been calculated using a common set of Harris parameters as it has also been presented for ${ }^{240} \mathrm{Pu}$ [3], where an alignment of octupole phonons with the rotational axis was discussed. As expected from a rotationalaligned octupole phonon [17], the alignment difference $\Delta i_{\mathrm{x}}$ between GSB and OCT is $\sim 3 \hbar$, see Fig. 3. Moreover, for the $\pi=+$ the alignment is close to $6 \hbar$ and close to $9 \hbar$ for the $\pi=-$ as expected from two-phonon and three-phonon octupole bands. Interestingly, both sets of states form an alternating-parity band with connecting $E 1$ transitions and the alternating sequence of $\pi=+$ and $\pi=-$ becomes yrast at around $J^{\pi}=10^{+}$before the particle alignment sets in at 
$\hbar \omega \approx 0.4 \mathrm{MeV}$. These sequences will be denoted by EXP (GSB,OCT) and EXP2 $(\pi=+,-)$ in the following.

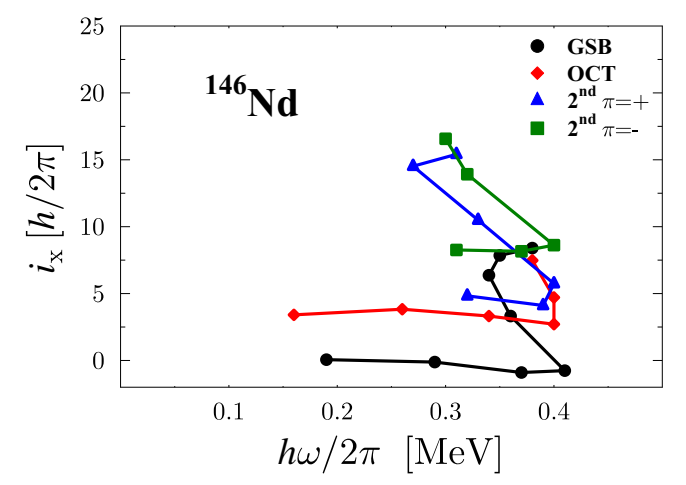

Figure 3. Alignment $i_{\mathrm{x}}$ with the rotational axis as a function of the rotational frequency $\hbar \omega$.

To further study this very interesting experimental observation the $s p d f$ IBM has been adopted and the signature splitting, defined as,

$$
S(J)=\frac{\left[E_{J+1}-E_{J}\right]-\left[E_{J}-E_{J-1}\right]}{E_{2_{1}^{+}}},
$$

has been studied in ${ }^{146} \mathrm{Nd}$. This quantity is a good measure for the realization of an alternating-parity band, i.e. $S(J) \approx 0$. The parameters of the spdf Hamiltonian, Eq. (2), were fixed by fitting them to the well-known lowlying collective excitations and their $\gamma$-decay properties.

$$
\begin{aligned}
\hat{H}_{s p d f}= & \epsilon_{d} \hat{n}_{d}+\epsilon_{p} \hat{n}_{p}+\epsilon_{f} \hat{n}_{f}-\kappa \hat{Q}_{s p d f} \cdot \hat{Q}_{s p d f} \\
& +a_{3}\left[\left(\hat{d}^{\dagger} \tilde{d}\right)^{(3)} \cdot\left(\hat{d}^{\dagger} \tilde{d}\right)^{(3)}\right]^{(0)}
\end{aligned}
$$

The number of negative-parity bosons $N_{p f}=\left\langle\hat{n}_{p}\right\rangle+$ $\left\langle\hat{n}_{f}\right\rangle$ was allowed to vary between 0 and 3 . We note that this choice of $\hat{H}_{s p d f}$ describes $s d$ and $p f$ states separately and does not mix positive-parity and negative-parity boson states, and thus provides a clear distinction.

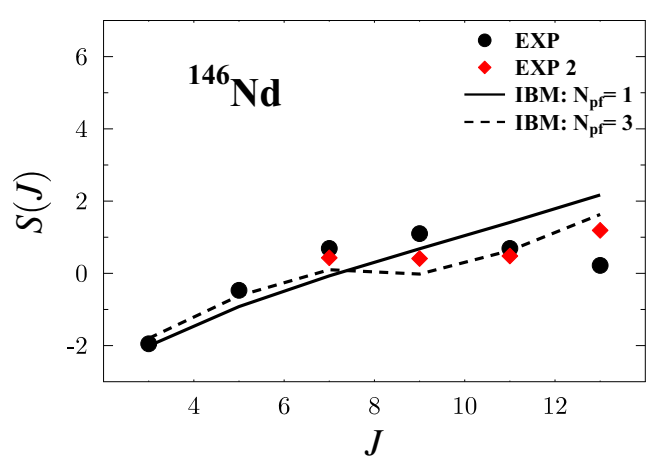

Figure 4. Experimental signature splitting $S(J)$ (symbols) compared with the IBM predictions (lines). Please note that only the yrast structure has been considered in the IBM calculations.
Figure 4 shows the experimental data in comparison with the model predictions. While the calculations with only one negative-parity boson do not reproduce the experimental signature splitting, especially in the region of the yrast structure change $\left(J^{\pi}=9^{-}, 10^{+}\right)$, the calculations allowing up to three negative-parity bosons follow closely the evolution of this quantity. At the same time, the two- and three-phonon octupole states have become yrast in the calculations at around $J^{\pi}=9^{-}, 10^{+}$, which furthermore agrees with the experimental band crossing of EXP and EXP2, and the observed alignments of the respective bands. Even though a finite-size effect because of the limited total boson number $N_{B}=6$ cannot be excluded, the present IBM calculations strongly support the interpretation of the four rotational bands as being of zero-, one-, two-, and three-phonon octupole character, respectively. Finally, we note, that this interpretation is in accordance with the condensation of rotational-aligned octupole phonons [17] and makes ${ }^{146} \mathrm{Nd}$ the first nucleus with candidates up to $n=3$.

This work was supported by the DFG (ZI 510/4-2) and the Romanian UEFISCDI Project No. PN-II-ID-PCE2011-3-0140. M.S. is supported by the Bonn-Cologne Graduate School of Physics and Astronomy.

\section{References}

[1] P.A. Butler and W. Nazarewicz, Rev. Mod. Phys. 68, 349 (1996)

[2] L.P. Gaffney et al., Nature 497, 199 (2013)

[3] X. Wang et al., Phys. Rev. Lett. 102, 122501 (2009)

[4] I. Wiedenhöver et al., Phys. Rev. Lett. 83, 2143 (1999)

[5] R.V. Jolos, P. von Brentano, and R.F. Casten, Phys. Rev. C 88, 034306 (2013)

[6] P.E. Garrett, J. Phys. G 27, R1 (2001)

[7] R.F. Casten et al., Phys. Lett. B 40, 333 (1972)

[8] I. Ragnarsson and R.A. Broglia, Nucl. Phys. A 263, 315 (1976)

[9] J.V. Maher et al., Phys. Rev. C 5, 1380 (1972)

[10] A. Friedman et al., Phys. Rev. C 9, 760 (1974)

[11] H.-F. Wirth et al., Phys. Rev. C 69, 044310 (2004)

[12] D.A. Meyer et al., Phys. Rev. C 74, 044309 (2006)

[13] M. Spieker et al., Phys. Rev. C 88, 041303(R) (2013)

[14] F. Iachello and A. Arima, The Interacting Boson Model (Cambridge University, Cambridge, England, 1987)

[15] N.V. Zamfir and D. Kusnezov, Phys. Rev. C 67, 014305 (2003)

[16] ENSDF, NNDC Online Data Service, ENSDF database, http://www.nndc.bnl.gov/ensdf(2014)

[17] S. Frauendorf, Phys. Rev. C 77, 021304(R) (2008)

[18] J.M. Hoogduin et al., Phys. Lett. B 384, 43 (1996) 
